\title{
UNSTEADY TRANSONIC FLOWS IN AN AXIAL FLOW COMPRESSOR*
}

BY

\author{
A. R. MANWELL
}

Cambridge, England

Introduction. This paper deals with some transonic flow problems for the supersonic axial flow compressor: these arise in connection with flutter analysis for fan blading. Very detailed solutions for unsteady plane transonic flows through a cascade of flat plates have been given by Goldstein Braun and Adamczyk (1977) and Nagashima and Whitehead (1977), see also Goldstein (1975), Kurosaka (1974), Mani and Horvay (1970), Miles (1959), Morfey and Fisher (1970) and Verdon and McCune (1975). The present paper treats the subject from the point of view of boundary value problems for the reduced wave equations of supersonic and subsonic flow, with particular reference to (a) the rotor in a finite duct and (b) a non-uniform incident stream.

In Secs. (1) and (2) we solve the supersonic entry problem for $\sigma=0, \pi$ by Fredholm theory. If the frequencies are small we can show that the solutions depend on the duct length, in some cases very significantly. The uniqueness proof of Sec. (3) requires strong conditions on the incident stream and, see App. (B), we give a counter-example for a differential equation of the same general class. If we could strengthen this result to do the same for a specific case of entry solutions in the two-dimensional model of the ducted rotor, (i.e. for Eq. (3.1) with given $\psi$ ) then we would have a basis for a strict analytical discussion of the 'buzz-saw' phenomenon, cf. Morfey and Fisher loc cit. Sec. (4) shows that the exit problems for the subsonic stream ought likewise to be set in a bounded region.

The above analysis of the differential equations has some interesting points of contact with the physical arguments of Ferri (1940), (1964) and Kantrowitz (1950). Ferri envisaged a supersonic biplane such that the external shock wave system might be eliminated. Kantrowitz, having developed a working supersonic compressor, explained its operation in terms of a two-dimensional model which is now standard, c.f. Levine (1957), Stratford and Sansome (1959), Hutton (1974) and Lichtfuss and Starken (1974), from an extensive literature.

\footnotetext{
* Received July 25, 1984.
} 
Kantrowitz reasoned that in order to attain a quasi-smooth steady entry flow, avoiding instability due to strong shocks extending far ahead of the rotor face, we need a precise balance between the weak shock compressions associated with the forward blade edges and the (simple wave) expansions over the rear blade segments. If this balance is not achieved then only the propagation of unsteady waves upstream can restore quasi-smooth flow. Ferri (1964), p. 387, goes further, proposing that the interference between the unsteady waves coming off successive blades produces changes in the axial component of velocity only: this is, in effect, condition (A) of Secs. (1), (2) below.

Because the physical flow problem involves such a fine balance the formulation of the boundary problem for the two-dimensional model needs careful study. In Secs. (1) (2) we propose three possibilities: either of these might prove most appropriate under various prescribed working conditions and each involves a finite entry region. The investigation of $(G)$, on the other hand, is based on the cascade in an unbounded stream. Here the uniqueness proofs, Manwell (1981) for the entry flows and Sec. (1) below for the flow behind the in-passage shock, certainly support the analysis. However, see App. (A) below, the necessary delicate balance has been achieved only by the introduction of a small dissipation and then, only in the strictly analytical sense. Difficulties arise as soon as we look into the effect of duct length or, again, non-uniformities in the entry flow. From the physical point of view it would therefore seem preferable to keep to the usual treatment of acoustic waves in a duct where end conditions are of basic importance.

1. The Kantrowitz model for supersonic entry flow; uniqueness proofs for supersonic and subsonic cascade flows. In the idealisation of flow into a ducted rotor which provides the two-dimensional cascade model, see Figs. (1) (2), we first suppose the rotor blades to be very stubby and consider a quasi-two-dimensional flow in a thin annular region bounded by circular cylinders each coaxial with the axis of the rotor. (This primitive model can be elaborated by 'strip theory'.) Thus we agree to ignore all radial motion, centripetal forces are not considered and the velocity along $O X$, see Fig. (2), corresponds to the axial component measured relative to the moving blade under consideration. Similarly, the velocity along $C C^{\prime}$ in the model corresponds to the 'swirling' component of the (unsteady) flow measured at the plane of entry to the duct. Ferri's hypothesis suggests that this latter component will be determined by the incident stream, or better, by the mean steady flow. We shall therefore set up a boundary value problem for slightly perturbed, usually unsteady, flows, prescribing (a) $\varphi$, the velocity potential along $C C^{\prime}$, (b) the velocity component normal to the blade segment and (c) the usual blade-to-blade periodicity (1.5) below. These conditions uniquely define the corresponding soluiions of the reduced wave equation for a uniform stream and this result can be extended to slightly non-uniform incident flows. We call this Problem (A).

There are, on a linear theory, other possible choices of the entry conditions at $C C^{\prime}$. For example we might fix the perturbation pressure and, compare $(\mathrm{G})$, Eq. (2.5) take as the independent variable

(B)

$$
p=\left(i \omega_{1}-\partial / \partial x\right) \varphi
$$


where $\omega_{1}$ is a frequency parameter. Again, there is a (formal) generalisation of the outgoing-wave condition of Sommerfeld, originally stated for the Helmholtz equation but often applied to the analysis of waves in subsonic flow. The analogue for entry conditions in the case of a finite duct would appear to be best expressed by setting $\tilde{\varphi}=0$ at $C C^{\prime}$ where, compare Copson (1975) p. 227 et seq also Courant and Hilbert (1962), Ch. IV, §5, we set

(C)

$$
\check{\varphi}=\left(i M_{1} \omega_{1}-\partial / \partial x\right) \varphi .
$$

Here $M_{1}>1$ denotes the Mach number of the entry flow and the right hand member is the usual Helmholtz expressions after the introduction of non-dimensional parameters. Here and in what follows the notation is that of $(G)$.

Let $\varphi_{1}, \varphi_{2}$ be solutions of the reduced wave equation as set in the region $\Omega: A B A^{\prime} C^{\prime} C$ and for a uniform supersonic entry flow. Then we have, with $\varphi_{1}-\varphi_{2}=\varphi$,

$$
\begin{aligned}
L[\varphi] & \equiv \varphi_{x x}-\varphi_{y y} / \beta_{1}^{2}-2 i M_{1} k_{1} \varphi_{x}-k_{1}^{2} \beta_{1}^{2} \varphi=0, \\
k_{1} \beta_{1}^{2} & =M_{1} \omega_{1},
\end{aligned}
$$

compare (G), Eq. (2.2). If we write $\psi=e^{-i M_{1} k_{1} x} \varphi$ we find

$$
\psi_{x x}-\psi_{y y} / \beta_{1}^{2}+k_{1}^{2} \psi=0 .
$$

Moreover not only $\varphi$ but both $p$ and $\check{\varphi}$ satisfy (1.3) and also the blade-to-blade periodicity condition

$$
\varphi^{\prime} \equiv \varphi\left(x^{\prime}, y^{\prime}\right)=e^{i \sigma} \varphi(x, y) ; \quad x^{\prime}=x+1, y^{\prime}=y+m ; m \beta_{1}<1 .
$$

which applies, in particular, between congruent points of the characteristics $A C, A^{\prime} C^{\prime}$. Again, the boundary conditions in the uniqueness proof, $\varphi=0$ at $C C^{\prime}$ and $\partial \varphi / \partial y=0$ at $A B$, respectively, go over to the same conditions on $\psi$ of Eq. (1.4); this extends to the analogous discussion based on (1.3) (1.4) for problems (B) and (C).

If we multipy (1.4) by $2 \bar{\psi}_{x}$ and apply Green's theorem in the region $\Omega$ this being bounded by $\Gamma: A B A^{\prime} C^{\prime} C A$ we get

$$
I=\oint_{\Gamma}\left\{\left(\left|\psi_{x}\right|^{2}+\left|\psi_{y}\right|^{2} / \beta_{1}^{2}+k_{1}^{2}|\psi|^{2}\right) d y+2 \mathbb{R} \psi_{x} \bar{\psi}_{y} / \beta_{1}^{2} d x\right\} .
$$

The contribution from $A B$ vanishes with $\varphi_{y}$ and for $B A^{\prime}$ we find

$$
I_{2}=\int_{B}^{A^{\prime}}\left(\left|\frac{d \psi}{d x}\right|^{2}+k^{2}|\psi|^{2}\right) d y \geqslant 0 .
$$

Also $\mathrm{I}_{3}=\int_{A^{\prime}}^{C^{\prime}}$ has a similar form and this cancels with $L_{5}=-\int_{A}^{C}$ because between corresponding points $\psi^{\prime} / \psi=\exp \left(i\left(\sigma+M_{1} k_{1}\right)\right)$. Finally, since $\varphi=0=\psi$ along $C C^{\prime}$ we get

$$
I_{4}=\int_{C}^{C^{\prime}} D\left|\psi_{y}\right|^{2} \beta_{1}^{-2} d y \geqslant 0 ; \quad D=1-m^{2} \beta_{1}^{2}>0 .
$$

It now follows that the non-negative contributions $L_{2}$ and $I_{4}$ each vanish and so both $\psi$ and $\partial \psi / \partial n$ vanish along $C C^{\prime}$. This ensures the vanishing of the solutions in the vicinity of 
the non-characteristic arc $C C^{\prime}$ and by using standard properties of hyperbolic equations also the periodic property (1.5) we see that it vanishes throughout the region $\Omega$. Finally the vanishing of the solution along $B A^{\prime}$ and the boundary condition at the blade segment $B D^{\prime}$ ensures that it vanishes in $\triangle B D^{\prime} A^{\prime}$.

To extend the proof to problem (B) we apply the previous arguments to show that $p$ vanishes in $\Omega$ and then observe that the potential must be of the form

$$
\varphi_{0}=e^{i \omega_{1} x}\left(a e^{\omega_{1} y}+b e^{-\omega_{1} y}\right)
$$

which cannot satisfy the periodicity condition and so must vanish. A similar argument starting with the condition $\check{\varphi}=0$ at $C C^{\prime}$ leads to the uniqueness proof for problem (C) and subject to $1<M_{1}<2$.

It is shown in $(\mathrm{G})$ that the unsteady flow behind the strong in-passage shock, that is to say the solution in the blade passages and behind the cascade, may be expressed in terms of $\Phi_{2}, \Psi_{2}$ where

$$
U_{2}=\left(\Phi_{2}\right)_{x}+\left(\Psi_{2}\right)_{y} ; \quad V_{2}=\left(\Phi_{2}\right)_{y}-\left(\Psi_{2}\right)_{x}
$$

and the problem can be reduced to determining $\Phi_{2}$ satisfying the reduced wave equation for subsonic flow and the following boundary conditions at the (normal) shock:

$$
U_{2}+h_{2} P_{2}=h_{1} P_{1} ; \quad\left(P_{2}\right)_{y}+h_{4}\left(\frac{\partial^{2}}{\partial y^{2}}-\omega_{2}^{2}\right) \Psi_{2}=h_{3} \frac{\partial P_{1}}{\partial y} .
$$

Here we have

$$
\begin{aligned}
& P_{2}=\left(i \omega_{2}-\partial / \partial x\right) \Phi_{2}, \quad h_{2}=\frac{1}{2}\left(1+M_{1}^{-2}\right), \\
& h_{4}=(\gamma+1)\left(M_{1} M_{2} / \beta_{1} \beta_{2}\right)^{2}, \quad M_{2}^{2}=1+\frac{\gamma-1}{2} M_{1}^{2} /\left(\gamma M_{1}^{2}-\frac{\gamma-1}{2}\right),
\end{aligned}
$$

the form of $h_{1}, h_{3}$ being immaterial in the present discussion. The potential function $\varphi=\Phi_{2}$ satisfies

$$
\begin{aligned}
\varphi_{x x}+\varphi_{y y} / \beta_{2}^{2}+2 i M_{2} k_{2} \varphi_{x}+k_{2}^{2} \beta_{2}^{2} \varphi & =0, \\
k_{2} \beta_{2}^{2} & =M_{2} \omega_{2}, \quad \beta_{2}^{2}=1-M_{2}^{2}
\end{aligned}
$$

and the boundary condition at the shock line may be written

$$
\begin{aligned}
& \left.\left(A\left(\Phi_{2}\right)_{x}+i B \omega_{2} \Phi_{2}\right)\right|_{0} ^{y}+\left.\omega_{2}^{2} \int_{0}^{y}(y-t)\left(\Psi_{2}\right)_{y}\right|_{y=t} d t=\cdots, \\
& A=1+h_{4}\left(1-k_{2}\right), \quad B=h_{2} h_{4}-1,
\end{aligned}
$$

where

$$
\left(\Psi_{2}\right)_{y}+\left(1-h_{2}\right)\left(\Phi_{2}\right)_{x}+i h_{2} \omega_{2} \Phi_{2}=\cdots
$$

and the right hand members are known from the entry solutions.

We now consider solutions of Eqn. (1.13) in the region $A D^{\prime} L L^{\prime}$ say,

$$
\tilde{\Omega}: x>0, \quad y>m(x-l), \quad 0<y<m \text {. }
$$


Setting $\phi=F+i G$ for the difference of two possible solutions we find

$$
\begin{aligned}
\oint\left(F G_{x}-G F_{x}\right. & \left.+M_{2} k_{2}\left(F^{2}+G^{2}\right)\right) d y \\
& +\left(G F_{y}-F G_{y}\right) d x / \beta_{2}^{2}=0 .
\end{aligned}
$$

If $\varphi_{y}$ vanishes at the blade segments and we also require $\varphi=0$ at the exit line $L L^{\prime}$ then, taking account of the periodicity between points of $y=0, y=m$ (or $y=2 m$ in case $\sigma=\pi$ ) the only contribution to (1.17) comes from the shock line(s). In the simplest case, if squares of $\omega_{2}$ may be beglected we get

$$
\omega_{2}\left(A M_{2}^{2}-B \beta_{2}^{2}\right) \int_{D^{\prime}}^{A^{\prime}}\left(F^{2}+G^{2}\right) d y=0
$$

and some straightforward reduction shows that the factor before the integral sign does not vanish provided $1<M_{1}^{2}<(3+\gamma) / 2$. The uniqueness proof follows from (1.14) showing that we have zero Cauchy data at the shock line. In general we treat (1.14) as a Volterra equation linking $\varphi_{x}$ with $\varphi$ and, after solving this, we get the same conclusion for sufficiently small values of $\omega_{2}$.

The numerical process of shock fitting as employed in $(\mathrm{G})$ is such as to suggest that some restriction on the magnitude of $\omega_{2}$ is probably necessary for the solution to be valid.

It will be observed that, in addition to the requirements of $(G)$ our uniqueness proof requires the prescription of one constant at the point $D$. We can avoid this by working with $\chi=\partial \varphi / \partial y$ rather than $\varphi$ but then we would have to prescribe $\chi$ instead of $\varphi$ along the exit line $L L^{\prime}$.

2. Construction of the solutions $\Phi_{1}$ for $\sigma=0, \pi$. To exhibit the dependence of the solutions on the choice of the entry line $C C^{\prime}$ we introduce a representation in terms of trigonometrical series. The first step is to define new coordinates $X, Y$ according to (2.1). Here, dropping the suffix on $\beta_{1}$, we set

$$
\begin{gathered}
D X=2\left(x-m \beta^{2} y\right), \quad D Y=2 \beta(y-m x), \\
D=1-m^{2} \beta^{2}>0
\end{gathered}
$$

where $D>0$ as before, and in view of the subsonic axial property. At $y=0$ we have $X=X(x)=2 x / D, Y=Y(X)=-2 m \beta x / D$ and with point $C(-\beta c, c)$ we find $Y(C)=$ $2 \beta c /(1-m \beta)$. We seek solutions for $\sigma=0$ or $\pi$ in the form

$$
\varphi=\sum_{n} \frac{c_{n} e^{c n \pi X}}{\mu_{n}-m \beta n \pi}\left(\exp \left(i \mu_{n} Y\right)-\exp \left(i\left(\overline{\mu_{n}-\lambda_{n}} Y(c)+\lambda_{n} Y\right)\right)\right),
$$

where $\operatorname{Sgn}(n)=\operatorname{sgn}(n)$ for $n \neq 0$ and $\operatorname{Sgn}(0)=-1$. Here we have

$$
\begin{aligned}
\lambda_{n}, \mu_{n} & =\frac{1}{2} M k m \beta \pm \operatorname{Sgn}(n), \Delta(n), \\
& =\frac{1}{2} M k m \beta \pm\left(n \pi-M \frac{k}{2}\right)+O\left(k^{2} / n\right), \\
\Delta(n)^{2} & =(n \pi-M k / 2)^{2}-{ }^{2} D / H,
\end{aligned}
$$


these estimates holding with respect to $k$ as $k$ tends to 0 with the single exception of $n=0$ which arises only for $\sigma=0$. (In the case $\sigma=\pi$ it is convenient to replace $n$ by $n+\frac{1}{2}$.) To avoid the case of equal roots in the indices we require $k<2 \pi /(M+1)$ for $\sigma=0$ and $k$ less than half this value for $\sigma=\pi$. The solution under (2.2) satisfies the periodicity condition and vanishes along $C C^{\prime}$. To satisfy the boundary condition at the blade segment we must have

$$
\begin{aligned}
\left.\frac{\partial \varphi}{\partial y}\right|_{y=0}= & \frac{2 i \beta}{D}\left\{\sum c _ { n } \left\{\exp \left(i\left(n \pi-m \beta \mu_{n}\right)\right) 2 x / D\right.\right. \\
& \left.+\Theta_{n} \exp \left(i\left(n \pi-m \beta \lambda_{n}\right)\right) 2 x / D\right\}, \\
= & W^{\prime}(x)-i \omega W(x),
\end{aligned}
$$

where

$$
\begin{aligned}
\left|\Theta_{n}\right| & =\left|\frac{\lambda n-m \beta n \pi}{m \beta n \pi-\mu_{n}} \cdot \exp \left(i\left(\mu_{n}-\lambda_{n}\right) Y(c)\right)\right| \\
& \leqslant \Theta=\frac{1-m \beta}{1+m \beta} .
\end{aligned}
$$

We write $f(\tilde{x})=\Sigma c_{n} \exp (\operatorname{in} 2 \tilde{x} \pi), \tilde{x}=x /(1-m \beta)$ and, using the asymptotic bounds of (2.3) to arrange (2.4) as an integral equation,

$$
\begin{aligned}
& f(\tilde{x})+\Theta f\left(\Theta x^{2}-Y(C)\right) \exp \{i M k(Y(C)+m \beta(1+\Theta) \tilde{x})\} \\
& =k \int_{0}^{1} K(\tilde{x}, t ; k \cdots) f(t) d t+g(x)
\end{aligned}
$$

where $K$ and $g$ are known; for $\sigma=\pi$ the factor $k$ before $K$ may be improved to $k^{2}$.

Regarding the right hand member as known (2.6) is of the form

$$
f(x)=k(x)-\Theta \exp [i(\lambda+\mu x)] f\left(x_{1}\right), \quad x_{1}=\Theta x-Y(C)
$$

and this, compare Picard (1927), is solved by the series

$$
\begin{aligned}
f(x)=h(x)- & \Theta \exp i(\lambda+\mu x) h\left(x_{1}\right) \\
& +\Theta^{2} \exp i\left(2 \lambda+\mu\left(x+x_{1}\right)\right) h\left(x_{2}\right), \ldots, \\
x_{n+1}=\Theta x_{n}- & Y(C) \ldots
\end{aligned}
$$

leading to a regular Fredholm equation equivalent to (2.6). Here $f$ is periodic and convergence is assured for $\Theta<1$. It should also be noted that the known function $g$ is given on a finite interval and the solution will, in general, have discontinuities in its first order derivatives across the characteristics $X=Y, X+Y=2 Y(C)(\bmod 2)$. However the uniqueness proof still applies and the solution of Eq. (2.6) and the corresponding boundary problem can be completed by using the Fredholm alternative.

In the case of rigid body motion of the blades we set, in our notation, the unit of length being $A B$,

$$
W=H+A(x-d)
$$


and define $Y^{*}$ as the positive non-integral part of $-Y(C)$. In case $0<\Theta+Y^{*}(C)<1$ the weak shock discontinuities reflected from $C C^{\prime}$ do not intersect the segment $A B$. Then, in case $\sigma=\pi$ we can solve (2.6) to order $\omega$ by simply equating coefficients and after setting $g(x)=f(x) \exp (-i M k x)$ with $g=g_{0}+g_{1} x$. We then find

$$
\begin{aligned}
& g_{0}=-\frac{D}{2 \beta(1+\Theta)}[i A /(1-m \beta)+\omega(H-A d)]+\frac{\Theta Y^{*} D A \omega\left(1+M^{2} / \beta^{2}\right)}{2 \beta(1+\Theta)\left(1+\Theta^{2}\right)}, \\
& g_{1}=-\frac{D A \omega\left[1+M^{2} / \beta^{2}\right]}{2 \beta\left(1+\Theta^{2}\right)} .
\end{aligned}
$$

It can then be shown that the velocity component along the blade is

$$
\begin{aligned}
\frac{\partial \varphi}{\partial x}= & \frac{1}{\beta} \frac{\partial \varphi}{\partial y}-\frac{2 i}{D}\{2 g(\tilde{x}) \exp i(M k \tilde{x}(1-m \beta)) \\
& +\frac{M^{2} \omega^{2}}{\beta^{2}}\left[(1-m \beta) \check{g}(\tilde{x})-\Theta(1+m \beta) \check{g}\left(\Theta \tilde{x}+Y^{*}\right)\right]+O\left(\omega^{2}\right), \\
\check{g}(\tilde{x})= & \frac{D A /(1-m \beta)}{2 \beta\left(1+\Theta^{2}\right)}\left(-\frac{1}{2}+\tilde{x}\right),
\end{aligned}
$$

and hence, neglecting $\omega^{2}$,

$$
\begin{array}{r}
\operatorname{Im}\left(\frac{\partial \varphi}{\partial x}\right)=\frac{\omega}{\beta}\left\{\frac{1-\Theta}{1+\Theta}(H-A d)+\frac{A Y^{*} \Theta}{1+\Theta}\left[\frac{M^{2}(1+m \beta)}{\beta^{2}(1-m \beta)}-\frac{2\left(1+M^{2} / \beta^{2}\right)}{1+\Theta^{2}}\right]\right\} \\
+0(x) .
\end{array}
$$

As shown in App. (A), the solutions with $\varphi$ set equal to zero are equivalent to the solutions of $(G)$ for the unbounded region. The introduction of the finite duct length modifies the solutions significantly. This is particularly striking if $W(x)$ vanishes near $x=0$ and the item under (2.12) and involving $Y^{*}$ does not vanish. Moreover, for fixed Mach number and blade geometry but variable $C C^{\prime}$, the correcting term has an oscillatory character, appearing and disappearing for changes of the duct length which are comparable with the blade chord. This feature, however, disappears from the first order solution if $M^{2}=$ $1 / 3 m^{2}+1 / 3$.

3. Non-uniform entry flows. The equation for acoustic waves in a non-uniform streaming supersonic flow may be written as

$$
\begin{gathered}
\left(\lambda \chi_{\xi}\right)_{\eta}+\left(\lambda \chi_{\eta}\right)_{\xi}+\left(\frac{\partial\left(\chi / a^{2}, \psi\right)}{\partial\left(\xi_{0} \eta\right)}+\frac{1}{a^{2}} \frac{\partial(\chi, \psi)}{\partial(\xi, \eta)}\right)_{t}+\rho \frac{J}{a^{2}} \chi_{t t}=0, \\
\lambda=\lambda(a), \quad J=\frac{\partial(x, y)}{\partial(\xi, \eta)}, \quad q^{2}=u^{2}+v^{2}, \quad a=a(q)
\end{gathered}
$$

see Manwell (1982). Here $\chi$ is the perturbation velocity potential, $\xi, \eta$ are characteristic coordinates for the steady flow and $\psi$ is the stream function for the latter. To find a 
non-trivial example of such a steady flow we seek simple wave solutions of the characteristic system

$$
\begin{gathered}
u_{\xi}=-T_{1} v_{\xi}, \quad y_{\xi}=T_{2} x_{\xi}, \\
u_{\eta}=-T_{2} v_{\eta}, \quad y_{\eta}=T_{1} x_{\eta}, \\
T_{1,2}=\tan (\theta+\varepsilon), \quad \varepsilon=\varepsilon(q) .
\end{gathered}
$$

compare Courant and Freidrichs (1948), Ch. IV B and in particular Fig. 20. For the present purposes it is sufficient to consider such solutions as

$$
\begin{gathered}
x=A(\xi)+B(\eta) C(\xi) \\
y=\int T_{2}(\xi) d A+T_{1}(\xi) B(\eta) C(\xi) \\
C^{\prime}(\xi) / C(\xi)=T_{1}^{\prime}(\xi) /\left(T_{2}-T_{1}\right)
\end{gathered}
$$

We first construct the solution between two parallel lines along which $\xi$ is constant as are also the quantities $u, v$ and $p$. The periodic solution may therefore be generated by replacing $(x, y)$ by $(x+1, y+m)$ and so on. Evidently the profile must be a concave/convex one, compare Lichtfuss and Starken (1974) pp. 56-61. A salient feature of such a simple wave solution is that it can be continued forward from the blade surface by only a finite distance before we encounter a shock line. From the standpoint of supersonic aerodynamice (the case $\omega=0$ ) we must expect some complications in the theory of boundary value problems and this seems to apply also in the case of acoustic waves.

We consider the case where $\Theta$, the inclination of the velocity vector to the main stream direction is small and we suppose that the axes are taken so that we have, with $T_{0}=\tan E$,

$$
x=A_{0} \xi+B_{0} \eta+\cdots, \quad y=T_{0}\left(B_{0} \eta-A_{0} \xi\right)+\cdots .
$$

Then equation (3.1) may be written as

$$
\begin{aligned}
& \chi_{\xi \eta}+r_{1} \chi_{\xi}+r_{2} \chi_{\eta}-c \frac{M k}{2}\left(\left(b_{0}+r_{3}\right) \chi_{\xi}+\left(q_{0}+r_{4}\right) \chi_{\eta}+r_{5} \chi\right) \\
& p_{0}=1+m \beta, \quad q_{0}=1-m \beta, \quad-\beta^{2} \frac{k^{2}}{4} p_{0} q_{0}\left(1+r_{0}\right) \chi=0
\end{aligned}
$$

the $r_{j}$ being small and having the periodic property with, say, $\sigma=0$. We set

$$
\chi=e^{i k\left(M / 2\left(q_{0} \xi+p_{0} \eta\right)+w(\xi, \eta)\right)} Z
$$

where $w$ satisfies

$$
w_{\xi \eta}+r_{1} w_{\xi}+r_{2} w_{\eta}+=\frac{M}{2}\left(r_{5}-q_{0} r_{1}-p_{0} r_{2}\right)
$$


and is defined by zero Cauchy data along $C C^{\prime}: \xi=\eta$. This leads to

$$
\tilde{\mathscr{L}}(Z) \equiv Z_{\xi \eta}+\tau_{1} Z_{\xi}+\tau_{2} Z_{\eta}+k_{1}^{2}\left(1+t_{0}\right) Z=0,
$$

with

$$
\begin{aligned}
\tau_{1} & =r_{1}+i k\left(\omega_{\eta}-\frac{1}{2} M r_{3}\right), \quad k_{1}^{2}=k^{2} p_{0} q_{0} / 4, \\
\tau_{2} & =r_{2}+i k\left(\omega_{\xi}-\frac{1}{2} M r_{4}\right),
\end{aligned}
$$

and $t_{0}$ real and small of the same order of magnitude as the $r_{j}$. From its construction $w(\xi, \eta)$ and hence $t_{0}$ also $|Z|$ retains the periodic property between $A C$ and $A^{\prime} C^{\prime}$.

We now extend the uniqueness theorem of Section 1 under the condition $\chi=0$ along $C C^{\prime}$, the periodic property between the characteristics and prescribed smooth values of $\partial \chi / \partial n$ along $A B$, the image of a rear blade segment in the $(\xi, \eta)$ plane. We first remark that by an elementary analysis based on (3.2), (3.3) the condition $\partial \chi / \partial n=0$ is equivalent to

$$
\chi_{\xi}-d \eta \chi_{\eta} / d \xi=0
$$

in the characteristic coordinates. We note also that, by a classical result, see Picard (1928), we may suppose that the characteristic coordinates are re-mapped (in the present case only slightly changed) so that the blade segment goes into $\eta=\Theta \xi+$ const and $C C^{\prime}$ remains on the line $\xi=\eta$. We now choose real multipliers $B, C$ in

$$
\mathscr{M}=2 B \bar{Z}_{\xi}+2 C \bar{Z}_{\eta}
$$

according to

$$
B=B_{0}+b_{1} \xi-b_{2} \eta ; \quad C=C_{0}-c_{1} \xi-c_{2} \eta .
$$

Here we first require

$$
B_{0}>C_{0} ; \quad c_{1}>0 ; \quad c_{2}>b_{1}>b_{2}>0 .
$$

Also since $\xi>\eta, 0<\eta<1$ holds for points of the closure of $\Omega$ we can, by taking the $b_{j}, c_{j}$ sufficiently small in comparison with $B_{0}, C_{0}$, arrange that

$$
B_{0} \leqslant B<B_{*}, \text { say, } C_{0} \geqslant C>C_{*}>0 .
$$

If we now multiply $\tilde{\mathscr{L}}(Z)$ by $\overline{\mathscr{M}}$, apply Green's theorem and take the real parts we get

$$
J_{0}+J_{1}+\sum_{j=1}^{5} I_{j}=0 \text {. }
$$

Here the area integrals are

$$
\begin{gathered}
J_{0}=\iint_{\Omega}\left(b_{2}\left|Z_{\xi}\right|^{2}+c_{1}\left|Z_{\eta}\right|^{2}\right. \\
\left.+\left\{k_{1}^{2}\left(1+t_{0}\right)\left(c_{2}-b_{1}\right)-\left(B \frac{2}{\partial \xi}+C \frac{\partial}{\partial \eta}\right) t_{0}\right\}|Z|^{2}\right) d \xi d \eta, \\
J_{1}=2 \iint_{\Omega} \mathbf{R}\left(\left(\tau_{1} Z_{\xi}+\tau_{2} Z_{n}\right)\left(B \bar{Z}_{\xi}+C \bar{Z}_{\eta}\right)\right) d \xi d \eta
\end{gathered}
$$


and the $I_{j}$ are the line integrals over $A B, B A^{\prime} \ldots$ taken in anti-clockwise order. It will be seen that having fixed $B, C$ as above the sum $J_{0}+J_{1}$ will be positive definite for sufficiently small $\left|\tau_{j}\right|$ and $\left|t_{0}\right|<1$. Then we have

$$
\begin{gathered}
I_{2}=\int_{B}^{A^{\prime}}\left(\left.C Z_{\eta}\right|^{2}+k_{1}^{2}\left(1+t_{0}\right) B|Z|^{2}\right) d \eta \geqslant 0, \\
I_{4}=\int_{0}^{1}\left\{B_{0}-C_{0}+\left(b_{1}+c_{1}+c_{2}-b_{2}\right) \xi\right\}\left|Z_{\xi}\right|^{2} d \xi \geqslant 0 .
\end{gathered}
$$

Also

$$
I_{1}=\int_{A}^{B}\left\{C\left|Z_{\eta}\right|^{2} d \eta-B\left|Z_{\xi}\right|^{2} d \xi-k_{1}^{2}\left(1+t_{0}\right)|Z|^{2}(C d \xi-B d \eta)\right\} .
$$

which, after use of (3.13), yields

$$
\begin{aligned}
I_{1}= & \int_{A}^{B}\left\{H_{1}-k^{2}\left(1+t_{0}\right)|Z|^{2}(C-\Theta B)\right\} d \xi, \\
H_{1}= & \frac{1}{4}(C / \Theta-B)\left|\frac{d \chi}{d \xi}\right|^{2}+k\left(B t_{2}-C t_{1}\right) \operatorname{Im}\left(\bar{\chi} \frac{d \chi}{d \xi}\right) \\
& +k^{2}\left(C \Theta t_{1}^{2}-B t_{2}^{2}\right)|\chi|^{2} .
\end{aligned}
$$

Again, in view of the periodicity of $w$ and $\chi$ we find

$$
\begin{array}{r}
I_{3}+I_{5}=\int_{C}^{A}\left\{\left(b_{1}-b_{2}\right)\left|Z^{\prime}(\xi, 0)\right|^{2}-k_{1}^{2}\left(c_{1}+c_{2}\right)\right. \\
\left.\times\left(1+t_{0}\right)|Z(\xi, 0)|^{2}\right\} d \xi .
\end{array}
$$

It is now an elementary, if slightly tedious matter, to show that the sum (3.24) with (3.25) can be cast in the form

$$
\mathscr{K}=\int\left\{\mathscr{H}_{2}(\xi) \Phi^{\prime}(\xi)^{2}-\mathscr{H}_{3} \Phi(\xi)^{2}\right\} d \xi,
$$

with $H_{2}(\xi)>0, \Phi(0)=0$ and $H_{3}$ small for nearly uniform flows. Then, by using the Schwarz inequality, we can show that $\mathscr{K}$ is non-negative for some small $k_{1}$. Hence, from (3.18) et seq the solutions $X$ and $Z$ vanish in $\Omega$.

The proof demands strong conditions. For example if $B, C$ are to be linear then (3.16) is necessary to ensure that $J_{0}, I_{4}$ also $I_{3}+I_{5}$ are non-negative and, at the same time, $C-\Theta B>0$ in (3.24) holds only if we make $c_{1}$ and $b_{1}$ small $0(1 / C A)$, as measured in the characteristic plane. Likewise, to ensure the positiveness of $J_{0}+J_{1}$, the same restriction must be imposed on $\left|\tau_{j}\right|, k$ and the $\left|r_{j}\right|$.

These observations, the great simplification of the preceding discussion for the special case of flat plates and the example of Appendix (B) suggests that uniqueness may not hold in general. However, it remains to be proved that this is so for any one member of (3.1).

Failure of uniqueness in the two-dimensional model, particularly for a very long entry region, might be linked with the general behaviour of disturbances in plane supersonic flow. Again, the 'buzzsaw' property of rotors would arise as a natural consequence of the indetermination of the strictly periodic property between blade and blade 
4. A property of the exit flows for $=0$. We consider periodic solutions of (1.13) in the series form

$$
\varphi=\sum_{n} \varphi_{n}=\sum\left\{a_{n} \exp \left(i n \pi \tilde{X}+\tilde{\lambda}_{n} Y\right)-b_{n} \exp \left(i n \pi \tilde{X}+\tilde{\mu}_{n} Y\right)\right\}
$$

Here we write

$$
\begin{aligned}
\tilde{D} \tilde{X} & =2\left(x+m \beta_{2}^{2} y\right), \quad \tilde{D} \tilde{Y}=2 \beta_{2}(y-m x), \\
\tilde{D} & =1+m^{2} \beta_{2}^{2}
\end{aligned}
$$

with

$$
\begin{aligned}
\tilde{\lambda}_{n}, \tilde{\mu}_{n} & =i M_{2} k_{2} R_{2} m \beta_{2} \pm|\tilde{\Delta}(n)|, \quad n \neq 0, \\
\tilde{\Delta}(n)^{2} & =\left(n \pi+M_{2} k_{2} / 2\right)^{2}-k_{2}^{2} \tilde{D} / 4, \\
k_{2}\left(M_{2}+\tilde{D}^{1 / 2}\right) & <2 \pi,
\end{aligned}
$$

and

$$
\bar{\chi}_{0}, \tilde{\mu}_{0}=i \frac{k_{2} \beta_{2}}{2}\left(m M_{2} \pm\left(1+m^{2}\right)^{1 / 2}\right) .
$$

Each term of (4.1) satisfies the periodicity condition for the case $\sigma=0$ and the series provides a complete set of solutions in the vicinity of $E E^{\prime}$, say $\tilde{Y}=0$.

We can arrange that $\varphi$ vanishes on $L L^{\prime}$ and then we find for $n \neq 0$,

$$
b_{n}=a_{n} \exp (-2|\tilde{\Delta}(n) \tilde{Y}(l, 0)|),
$$

showing that the $b_{n}$ are uniformly small for large $|l|$. We note also that

$$
\mathscr{D}_{n} \varphi_{n}(\tilde{X}, 0)=\frac{\partial \varphi_{n}}{\partial \tilde{Y}}(\tilde{X}, 0)
$$

with

$$
\mathscr{D}_{n}=|\tilde{\Delta}(n)| \operatorname{coth}|\bar{\Delta}(n) \tilde{Y}(l, 0)|+i \frac{M_{2} k_{2} m}{2} \beta_{2}
$$

and

$$
\mathscr{D}_{0}=k_{2} \beta_{0}\left\{\left(1+m^{2}\right)^{1 / 2} \cot \left|\frac{k_{2} \beta_{2}}{2}\left(1+m^{2}\right)^{1 / 2} \bar{Y}(l, 0)\right|+i M_{2} m\right\} \text {. }
$$

An elementary observation is that for a whole sequence of values of $\tilde{Y}(l, 0)$ the solution $\varphi_{(0)}$ vanishes along both $E E^{\prime}$ and $L L^{\prime}$.

We now show how, in principle, the boundary value problem for the solution $\Phi_{2}$ set in $\tilde{\Omega}$ may be reduced to an auxiliary problem set in the blade passage $\tilde{\Omega}_{1}: A^{\prime} D^{\prime} E E^{\prime}$. The first step is to use the Fourier series expansions for $\varphi, \varphi_{\tilde{Y}}$ noting (4.7), to write down the relation, based on the solution in $\tilde{\Omega}_{2}$,

$$
\varphi(\tilde{X}, 0)=\int L_{1}(\tilde{X}, t ; l) \varphi_{y}(t, 0) d t
$$

which holds for general Cauchy data along $E E^{\prime}$. Then a simple modification of the uniqueness proof of (1.13) et seq shows that the corresponding problem set in $\tilde{\Omega}_{1}$ is 
uniquely defined by the original conditions also values of $\varphi$ along the boundary line $E E^{\prime}$. The actual construction of the solutions requires a fairly lengthy use of integral equations. However, it is reasonable to assume that, as in the case of Laplace's equation, (to which equation (1.13) tends for small values of the frequency parameter) we can find a second relation connecting $\varphi, \varphi_{y}$ with the data, and that this is of the form

$$
\varphi(\tilde{X}, 0)=\int L_{2}(\tilde{X}, t ; l) \varphi_{y}(t, 0) d t+g(\bar{X})
$$

Combining (4.9) and (4.10) we get another linear relation to determine $\varphi_{y}$ and so the values of $\varphi$ along $E E^{\prime}$. The significant observation is that because of the possible item in $\varphi_{(0)}$ and equation (4.8) the kernel of (4.9) contains a periodic item. Hence even for the exit flows it would be very surprising if the solutions tend smoothly into an asymptotic value for $l \rightarrow \infty$.

Acknowledgement. I would like to thank Dr D. S. Whitehead for drawing my attention to the combination tone which characterises supersonic rotors.

Appendix A. We show that if we admit a very small dissipative term in the reduced wave equation then the solutions which are bounded at infinity in the upstream direction are precisely those which result from dropping the $\Theta$-terms in (2.4) et seq. The modified equation is

$$
L[\varphi]+\varepsilon\left(i \omega_{1}-\frac{\partial}{\partial x}\right) \nabla^{2} \varphi=0
$$

compare Lamb (1952) p. 328, equations 4 , also p. 359. If $\varepsilon$ is very small we may replace $\varphi$ in the correction terms by $\varphi_{\lambda_{n}}$ or $\varphi_{\mu_{n}}$ as the case may be and an easy calculation gives the small perturbations of the indices according to

$$
2|\Delta(n)| \operatorname{Sgn}(n) \delta\left(i \lambda_{n}\right)=\frac{\varepsilon(\lambda n, n)}{D}\left(2 n \pi-2 m \beta_{1} \lambda_{n}-\omega_{1} D\right) .
$$

It then follows from a check of the signs that the $\lambda_{n}$-functions of equation (2.2) increase exponentially as $Y$ tends to infinity and so must be excluded from the series solution. To show that the other functions decrease it is sufficient to show that the product of the last factor on the right of (A.2) with the corresponding item involving $\mu_{n}$ remains positive with

$$
D\left(\left(2 n \pi-\omega_{1}\right)^{2}+k_{1}^{2} m^{2} \beta_{1}^{4} / M_{1}^{2}\right) .
$$

In a slight re-arrangement of this discussion we note also that for fixed $(x, y)$ the terms of the second series on the right of (2.2) tend to zero as $c, Y(C)$ increase without limit.

Formally the same conclusions may be derived by the damping device of $(\mathrm{G})$ which leads to (A.2) as before, although, strictly speaking, the $\varepsilon$ are different for each $n$.

Appendix B. Equations for which Problem A has eigenfunctions. Let $\varphi$ be any solution of (1.3) subject to the periodicity condition (1.5) with $\sigma=0$ and suppose $\varphi$ vanishes on $C C^{\prime}$. Let

$$
\varphi=e^{k w} \psi
$$

and suppose $w$ satisfies

$$
w(x+1, y+m)=w(x, y)
$$


for points of $A^{\prime} C^{\prime}, A C$, respectively. Then $\psi$ satisfies

$$
\begin{gathered}
L(\psi)+2 k\left(w_{x} \psi_{x}-w_{y} \psi_{y} / \beta^{2}\right) \\
+\psi\left(k L_{0}(w)+k^{2}\left(w_{x}^{2}-w_{y}^{2} / \beta^{2}\right)\right)=0,
\end{gathered}
$$

where

$$
L_{0}(w)=w_{x x}-w_{y y} / \beta^{2}-2 i M k w_{x} .
$$

If, in addition, $w$ satisfies the following

$$
\varphi_{y} / \varphi=k w_{y}, 0<x<1-m \beta ; y=0
$$

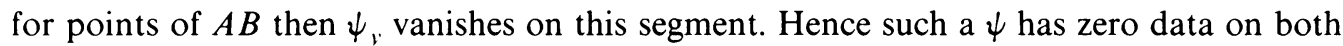
$C C^{\prime}$ and $A B$ and satisfies the periodic condition between congruent points of $A^{\prime} C^{\prime}$ and $A C$ : it is, therefore, an eigenfunction for Eq. (B.3) with the boundary conditions of Problem A.

The simplest choice is to take $n=0$ in (2.2) and then (B.5) gives

$$
\begin{aligned}
& w_{y}(x, 0)=\frac{\beta}{D}\left\{M m \beta+i \beta\left(1+m^{2}\right)^{1 / 2} \cot \mathscr{A}\right\} ; \\
& \mathscr{A}=k \beta\left(1+m^{2}\right)^{1 / 2}(\beta c /(1-m \beta)+m \beta x / D) .
\end{aligned}
$$

Here we choose $k, c$ according to

$$
k c \beta^{2}\left(1+m^{2}\right)^{1 / 2}=\frac{\pi}{2}(1-m \beta)
$$

and suppose that $k$ is small making $c$ large $O(1 / k)$. If also $L_{0}(w)=M^{2} k^{2} w$ we have solutions

$$
w=\exp (i M k x)\{f(x+\beta y)+i g(x+\beta y)\}
$$

and we will take $f(0)=g(0)=0$ the derivatives of $f$ and $g$ being determined by (B.6) for $0<x<1-m \beta$ and remaining disposable in $1-m \beta<x<1+m \beta$. Hence we can satisfy the periodicity condition between congruent points of $A C: x+\beta y=0$ and $A^{\prime} C^{\prime}$ : $x+\beta y=1+m \beta$.

Some routine calculations give

$$
\begin{gathered}
w_{x}=i M m \beta \exp (-i M k \beta y)+O(m k), \\
w_{y}=\beta w_{x}+O(m k), \\
w_{x}^{2}-w y^{2} / \beta^{2}=O(k),
\end{gathered}
$$

from which it follows that we may write B3 in the form

$$
\begin{aligned}
\psi_{x x} & -\psi_{y y} / \beta^{2}-2 c M k(1+O(m)) \psi_{x} \\
& +O\left(m k \psi_{y}\right)-\beta^{2} k^{2} \psi(1+O(k))=0,
\end{aligned}
$$

as required.

It should be added that this example, taken with Sec. (3), certainly suggests that the entry problem for a long duct is badly set but we must remember that we are working with complex solutions and have not shown that any specific member of the (very wide) class of equations (3.1) has eigenfunctions for the problems under consideration. 


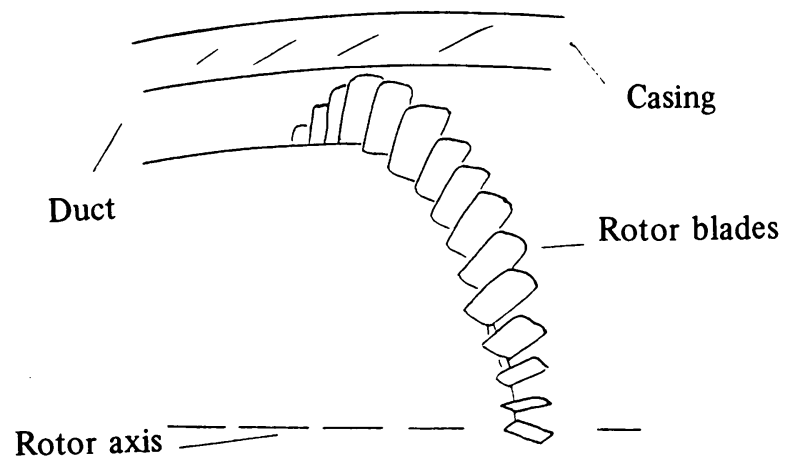

FIgURE 1: Supersonic compressor (after Kantrowitz)

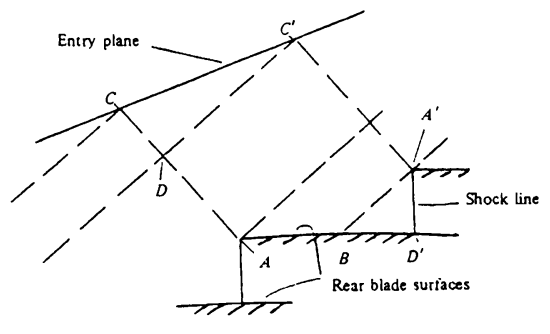

Mach lines - - . Rotation: $\overrightarrow{C^{\prime} C}$

FIGURE 2: Flow plane for supersonic entry

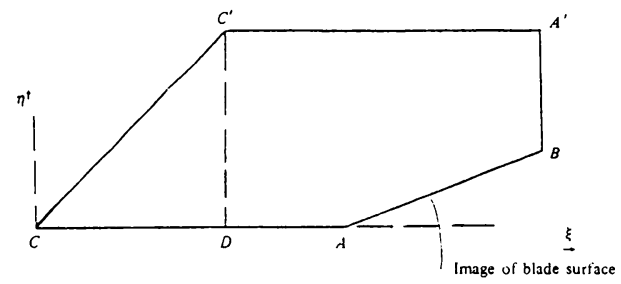

Figure 3: Characteristic plane

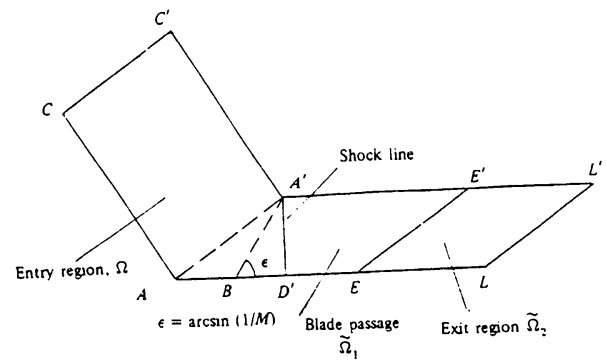

FIgURE 4: Scheme for transonic flutter analysis 


\section{REFERENCES}

Copson, E. T., Partial differential equations, Cambridge, 1975

Courant, R. and Friedrichs, K. O., Supersonic flow and shock waves, 1948 Interscience

Courant, R and Hilbert, D., Methods of mathematical physics, Vol. II, Interscience, 1962

Ferri, A., Esperienze su di un biplano iperacustica tipo Busemann, Atti di Guidonia No. 37-38, 1940

Ferri, A., Aerodinamic properties of supersonic compressors, High Speed Aerodynamics and Jet Propulsion, Vol. X (Edit. W. R. Hawthorne) Princeton, 1964

(joldstein, M. E., Cascade with subsonic leading-edge locus, AIAA Journ. 131117 (1975)

Goldstein, M. E., Braun, W. and Adamczyk, J. J., Unsteady flow in a supersonic cascade with strong in-passage shocks. J. Fluid Mech. 83, 569 (1977)

Hutton, M. F., Supersonic inflow onto turbine blading: the unique incidence theorem, Gen. Elect. Gen Board Res. Rept. R/M/M 159, 1974

Kantrowitz, A.. The supersonic axial-flow compressor, N.A.C.A. Tech. Rep. no. 974, 1950

Kurosaka, M., On the unsteady supersonic cascade with a subsonic leading edge- an exact first order theory-Part I. Trans. ASME, A.96 13(1974)

Lamb, R., Hydrodynamics (6th ed.)Cambridge, 1952

Levine, P., Two-dimensional inflow conditions for a supersonic compressor with curved blades. J. Appl. Mech. 24, 165 (1957)

Lichtfuss, H-J and Starken, H., Supersonic cascade flow, Progress in Aerospace Sciences 15, Edit. D. Kuchemann, Pergamon, 1974

Mani, R. and Horvay, G., Sound transmission through blade rows, J. Sound Vib. 12, 59 (1970)

Manwell, A. R., A uniqueness theorem for the moving medium reduced wave equation. Appl. Anal. 12, 215 (1981)

Manwell, A. R., Supersonic cascade flows with subsonic axial Mach number, Univ. Camb. Eng. Dept. Rep. CUED/A-Turbo/TR110, 1982

Miles, J. W., The potential theory of unsteady supersonic flow, Cambridge

Morfey. C. L. and Fisher, M. J., Shock wave radiation from a supersonic ducted rotor, Aer. Journ. 74, 579 (1970)

Nagashima, T and Whitehead, D. S., Linearized supersonic unsteady flow in cascades, Aer. Res. Coun. R \& M No. 3811,1977

Picard, Ė, Leçons sur quelque équations aux dérivée partielles, Paris, Gauthier-Villars, 1927

Picard, É, Leçons sur quelque équations fonctionelles, Paris, Gauthier-Villars, 1928

Stratford, B. S. and Sansome, G. E., The performance of supersonic turbine muzzles, Aer. Res. Coun. R \& M No. 3273,1959

Verdon, J. M. and McCune, J. E., Unsteady supersonic cascade in subsonic axial flow, AIAA Journ. 13, 193 (1975) 